\title{
Comparison of the synergistic effect of lipid nanobubbles and SonoVue microbubbles for high intensity focused ultrasound thermal ablation of tumors
}

Yuanzhi Yao, Ke Yang, Yang Cao, Xuan Zhou, Jinshun Xu, jianxin Liu, Qi Wang, Zhigang Wang, Dong Wang

Microbubbles (MBs) are considered as an important enhancer for high intensity focused ultrasound (HIFU) treatment of benign or malignant tumors. Recently, different sizes of gas-filled bubbles have been investigated to improve the therapeutic efficiency of HIFU thermal ablation and reduce side effects associated with ultrasound power and irradiation time. However, nanobubbles (NBs) as an ultrasound contrast agent for synergistic therapy of HIFU thermal ablation remain controversial due to their small nano-size in diameter. In this study, phospholipid-shell and gas-core NBs with a narrow size range of 500 to $600 \mathrm{~nm}$ were developed. The synergistic effect of NBs for HIFU thermal ablation was carefully studied both in excised bovine livers and in breast tumor models of rabbits, and made a critical comparison with that of commercial SonoVue microbubbles (SonoVue MBs). In addition, the pathological changes of the targeted area in tumor tissue after HIFU ablation were further investigated. Phosphate buffer saline (PBS) was used as the control. Under the same HIFU parameters, the quantitative echo intensity of B-mode ultrasound image and the volume of coagulative necrosis in lipid NBs groups were significantly higher and larger than that in PBS groups, but could not be demonstrated a difference to that in SonoVue MBs groups both ex vivo and in vivo. These results showed that the synergistic effect of lipid NBs for HIFU thermal ablation were similar with that of SonoVue MBs, and further indicate that lipid NBs could potentially become an enhancer for HIFU thermal ablation of tumors . 
1 Comparison of the synergistic effect of lipid nanobubbles and SonoVue microbubbles for 2 high intensity focused ultrasound thermal ablation of tumors

3

4

6

Yuanzhi Yao ${ }^{1}$, Ke Yang 2 , Yang Cao ${ }^{1}$, Xuan Zhou ${ }^{3}$, Jinshun $\mathrm{Xu}^{1}$, Jianxin Liu1, Qi Wang ${ }^{4}$, Zhigang Wang ${ }^{1 *}$, Dong Wang ${ }^{1,2 * *}$

${ }^{1}$ Chongqing Key Laboratory of Ultrasound Molecular Imaging, the Second Affiliated Hospital of Chongqing Medical University, Chongqing, 400010, PR China

2.Department of Ultrasound, Children's Hospital of Chongqing Medical University, Chongqing, 400014, PR China

3.Department of Emergency, Chinese PLA General Hospital, Beijing, 100853, PR China

4.Institute of Ultrasound Engineering in Medical of Chongqing Medical University, Chongqing, 40016, PR China

Corresponding authors

Dong Wang

Department of Ultrasound, Children's Hospital of Chongqing Medical

University, Chongqing, 400014, PR China

wangketongtong@163.com

Zhigang Wang

Chongqing Key Laboratory of Ultrasound Molecular Imaging, the Second Affiliated Hospital of Chongqing Medical University, Chongqing, 400010, PR China

wzg62942443@163.com 


\begin{abstract}
Microbubbles (MBs) are considered as an important enhancer for high intensity focused ultrasound (HIFU) treatment of benign or malignant tumors. Recently, different sizes of gasfilled bubbles have been investigated to improve the therapeutic efficiency of HIFU thermal ablation and reduce side effects associated with ultrasound power and irradiation time. However, nanobubbles (NBs) as an ultrasound contrast agent for synergistic therapy of HIFU thermal ablation remain controversial due to their small nano-size in diameter. In this study, phospholipid-shell and gas-core NBs with a narrow size range of 500 to $600 \mathrm{~nm}$ were developed. The synergistic effect of NBs for HIFU thermal ablation was carefully studied both in excised bovine livers and in breast tumor models of rabbits, and made a critical comparison with that of commercial SonoVue microbubbles (SonoVue MBs). In addition, the pathological changes of the targeted area in tumor tissue after HIFU ablation were further investigated. Phosphate buffer saline (PBS) was used as the control. Under the same HIFU parameters, the quantitative echo intensity of B-mode ultrasound image and the volume of coagulative necrosis in lipid NBs groups were significantly higher and larger than that in PBS groups, but could not be demonstrated a difference to that in SonoVue MBs groups both ex vivo and in vivo. These results showed that the synergistic effect of lipid NBs for HIFU thermal ablation were similar with that of SonoVue MBs, and further indicate that lipid NBs could potentially become an enhancer for HIFU thermal ablation of tumors.
\end{abstract}

\title{
Introduction
}

High intensity focused ultrasound (HIFU) has been gained widespread attention in research and application of tumor treatment (Hassanuddin et al., 2014; Peek et al., 2015; Zhang et al., 2011). Owing to the true noninvasiveness, availability and economic benefit in clinical practice, HIFU has achieved rapid development in the treatment of benign and malignant solid tumors in breast, prostate, liver and pancreas tissue in the past years (Cavallo et al., 2015; Uchida et al., 2012; Kazarian et al., 2008). Even more exciting is that HIFU thermal ablation therapy has already been FDA-approved for treating uterine fibroids in United States (Hesley et al, 2013). However, although the development of HIFU for tumor treatment was quite inspiring, HIFU is still restricted by its intrinsic limitations for large tumors (Zhou, 2011). The HIFU treatment time is currently on the order of hours and lesions formed by single HIFU exposure are fairly small (several to dozen $\mathrm{mm}^{3}$ ). For example, to achieve a large volume of tumors destruction hundreds of HIFU exposure, hours of treatment and / or higher ultrasound power is required (Fischer et al, 2010). However, side effects including skin burns and unintended heating to healthy tissue are inevitable as a result of long treatment time and high ultrasound power required for continuous lesion formation. Therefore, in order to overcome its intrinsic limitations, one strategy is to improve the therapeutic transducer based on multiple elements transducer using 
fast electronic-steering phased array transducer, which belongs to the HIFU engineering field (Ellens et al., 2015). Another strategy to accelerate the therapeutic efficiency of HIFU is to introduce the enhancer into the targeted region during HIFU exposure (Moyer et al., 2015; Sun et al., 2012; Hamano et al., 2014; Ma et al., 2014).

At present, different sizes of gas-filled bubbles were introduced into the targeted region to enhance the therapeutic efficiency of HIFU thermal ablation and reduce side effects through changing the acoustic property of the targeted tissue, resulting in the ultrasound energy accumulation in the intended target region to damage the tissue (Hamano et al., 2014; Ma et al., 2014; Zhang et al., 2014; Zhou et al., 2015). Micrbubbles (MBs) are well known to be an important enhancer for synergistically and extensively accelerating the lesion formation of ultrasound-mediated heating and cavitation activity during HIFU treatment procedure (Luo et al., 2006; Peng et al., 20012). In addition, HIFU has the potential of inducing anti-tumor immune response, and simultaneously bubbles could remarkably improve the anti-tumor immune response ( $\mathrm{Wu}$ et al., 2004; Liu et al., 2012). Bubbles in the high acoustic pressures will experience nonlinear oscillation known as inertial cavitation, and radiate out of higher frequencies ultrasound with massive energy, which are more readily absorbed by tissues and availably converted into heat to damage the tumor cells (Coussios et al., 2007). MBs serving as cavitation nuclei in the targeted area could lower cavitation thresholds and enhance tissue heating during HIFU treatment (Tran et al., 2005; McDannold, Vykhodtseva \& Hynynen, 2006). However, owing to MBs rapidly disappearing from circulation, multiple infusions during HIFU treatment are required. Additionally, because of MBs too large in size, multiple infusions in a time are risk of gas embolism. In order to overcome these limitatios of MBs, phase-shift nanodroplets are being now developed as a cavitation nucleation agent to enhance HIFUmediated thermal ablation (Kopechek et al., 2014; Zhang et al., 2011). Many of the liquid perfluorocarbon droplets in previous studies composed of relatively high boiling-point perfluorocarbons in order to achieve stability, but which required more acoustic energy to induce vaporization (Rapoport et al., 2011; Kopechek et al., 2014). Meanwhile, the generated gas bubbles from liquid perfluorocarbon droplets will easily coalesce into large ones to occlude arterioles, causing unwanted arterial occlusion, ischemia and potentially tissue infarction. Hence, the safety of these liquid perfluorocarbon droplets in the body needs further investigation.

With the development of nanotechnology, nanobubbles (NBs) with various shells (protein, polymers and phospholipids) have increasingly attracted more attention in ultrasound molecular imaging and disease therapy (Huang et al., 2013). Based on several previous studies (Yin et al., 2012; Fan et al., 2013), phospholipid-shell and gas-core NBs have shown optimal imaging abilities, high stability and easy penetrability of tumor vessels. Consequently, NBs may potentially become a good alternative to MBs and/or liquid perfluorocarbon droplets in the ultrasound therapy. Moreover, current research on NBs mostly focuses on ultrasound molecular imaging and drug or gene carriers (Yin et al., 2012; Xie et al., 2015; Cavalli, Bisazza \& Lembo, 2013). The research of NBs in synergistic HIFU thermal ablation is still in its initial stages (Zhang et al., 2014; Wang et al., 2012). In this study, we would like to further introduce lipid NBs and SonoVue MBs into HIFU treatment procedure to evaluate the effect of lipid NBs for 
synergistic HIFU ablation.

Herein, we introduced lipid NBs and Sulfur hexafluoride MBs (SonoVue, routinely used in clinic) to enhance HIFU thermal ablation and explore the synergistic effect of lipid NBs and SonoVue MBs in excised bovine liver and in vivo breast tumor models of rabbits during HIFU treatment procedure. NBs were successfully fabricated with phospholipids shell materials similar to those of sulfur hexafluoride microbubbles. Firstly, we compared the echo intensity of B-mode ultraosund image and the volume of the necrotic tissues induced by diverse HIFU parameters combined with lipid NBs or SonoVue MBs in ex vivo bovine liver. Then, we equally made a comparison in rabbits VX2 breast tumor models, and detected the pathological change after HIFU thermal ablation using hematoxylin-eosin (HE) staining and transmission electron microscope (TEM) and examined the proliferation of tumor cells using immunohistochemistry.

\section{Materials and methods}

\section{Preparation of NBs and MBs}

The lipid NBs were fabricated as described previously (Wang et al., 2010). Briefly, $5 \mathrm{mg}$ 1,2-dipalmitoyl-sn-glycero-3-phosphocholine (DPPC) and $2 \mathrm{mg}$ 1,2-dipalmitoyl-sn-glycero-3phosphoethanolmine (DPPE) were mixed in a vial (actual volume $1.5 \mathrm{ml}$ ), then $450 \mu$ phosphate buffer saline (PBS) and 50 $\mu$ l glycerol were added to prepare suspension, and then the suspension was incubated in a water bath at $45{ }^{\circ} \mathrm{C}$ for $30 \mathrm{~min}$. The vial was capped with a rubber cap, and then the air in the vial was replaced with perfluoropropane $\left(\mathrm{C}_{3} \mathrm{~F}_{8}\right)$ gas. Finally, the suspension was mechanically vibrated at $4000 \mathrm{rpm}$ for $90 \mathrm{~s}$. The purified lipid NBs were separated from the prepared bubbles with various diameters using a low-speed centrifugation (300 rpm, $3 \mathrm{~min}$ ) and a higher-speed centrifugation $(800 \mathrm{rpm}, 5 \mathrm{~min})$ for three times. The purified lipid NBs were finally resuspended in $3 \mathrm{ml} \mathrm{PBS}$ and stored at $4{ }^{\circ} \mathrm{C}$ for further use.

The MBs used in this study were commercial sulfur hexafluoride microbubbles (SonoVue, Bracco, Italy), which were approved for routine use in clinical ultrasonography in China. $5 \mathrm{ml}$ sterile saline was added into the vial before use to form a liquid solution by vigorous vibration.

The particle sizes of lipid NBs and SonoVue MBs were measured using dynamic light scattering (Zeta SIZER3000HS; Malvern, UK). Fresh bubbles were prepared for each experiment. Meanwhile, the concentration of lipid NBs and SonoVue MBs were also counted with globulimeter (Yangling, Jiangsu, China) according to the calculation guidelines.

\section{Animal models}

All the rabbits in this study, weighing about $2.0 \sim 2.5 \mathrm{~kg}$, were purchased from the Animal Center of Chongqing Medical University. The VX2 tumor was obtained from the laboratory of Ultrasound Engineering Institute of Chongqing Medical University. All animal experimental procedures were approved by the Animal Ethics Committee of Chongqing Medical University (Approval Number SYXK (Chongqing) 2012-0001).

Under sterile conditions, the VX2 tumor tissue was cut into small pieces (about 0.5 1.0 mm) using ophthalmic scissors and then cultivated in the mammary tissue of rabbits underneath the 
165

166

167

168

169

170

171

172

173

174

175

176

177

178

179

180

181

182

183

184

185

186

187

188

189

190

191

192

193

194

195

196

197

198

199

200

201

202

203

204

205

second bilateral nipples (Sun et al., 2012). To prevent infection, 800,000 units of penicillin were intramuscularly injected for three days.

\section{Ex vivo excised bovine liver ablation by HIFU}

The JC-200 focused ultrasound system (Chongqing Haifu Technology, Chongqing, China) was used as described previously (Wang et al., 2012). The system mainly consists of therapeutic ultrasound unit, diagnostic ultrasound unit and a central processing system. The therapeutic transducer has a focal length of $140 \mathrm{~mm}$, a diameter of $220 \mathrm{~mm}$ and a working frequency of 0.94 MHz. The focal region is $9.8 \mathrm{~mm}$ along the beam axis and $1.3 \mathrm{~mm}$ in the transverse direction. The diagnostic transducer with center frequencies of $3.5-5 \mathrm{MHz}$ was installed in the center of the therapeutic transducer and moved together to guide and monitor the treatment procedure in real time. The integrated transducers are submerged in degassed water.

Fresh bovine liver tissues were purchased from local abattoir and used within $12 \mathrm{~h}$ after slaughter. The liver was sliced into $12 \mathrm{~cm} \times 6 \mathrm{~cm} \times 6 \mathrm{~cm}$ in size, and immersed into a normal saline and degassed at $37^{\circ} \mathrm{C}$ for $30 \mathrm{~min}$. Lipid NBs $\left(200 \mu \mathrm{l}, 1.0 \times 10^{5}\right.$ bubbles $\left./ \mathrm{ml}\right)$ were directly injected into the targeted area using a syringe and monitored by the diagnostic ultrasound unit. After injection, HIFU thermal ablation was immediately performed on the injection site with diverse HIFU parameters ( $120 \mathrm{~W}$ for $5 \mathrm{~s} ; 150 \mathrm{~W}$ for $5 \mathrm{~s} ; 180 \mathrm{~W}$ for $5 \mathrm{~s}$ ). Similarly, SonoVue MBs $\left(200 \mu \mathrm{l}, 1.0 \times 10^{5}\right.$ bubbles $\left./ \mathrm{ml}\right)$ and PBS $(200 \mu \mathrm{l}, \mathrm{pH}=7.4)$ were employed for comparison under the same HIFU parameters. Before and after HIFU exposure, the echo intensity of B-mode ultrasound image within region of interest (ROI) was automatically calculated using Gray Val 1.0 Software affiliated to HIFU system. Each group was repeatedly carried out eight times. The mean echo intensity for each ROI was calculated separately in this program. The average value of the eight ROIs echo intensities were adopted as the mean echo intensity of the group. After HIFU thermal ablation, the length, width and depth of necrotic tissues were measured to calculate their volumes in the bovine liver using the following formula: $\mathrm{V}=\pi / 6 \times \mathrm{L} \times \mathrm{W} \times \mathrm{D}$ ( $\mathrm{L}$ : length, W: width, D: depth).

\section{In vivo HIFU thermal ablation of rabbit breast VX2 tumors}

Three weeks after implantation of tumors, the rabbit breast VX2 tumors (about $10 \mathrm{~mm}$ in diameter) were carefully depilated with $8 \% \mathrm{Na}_{2} \mathrm{~S}$ again. After anesthetization using 3\% pentobarbital sodium $(30 \mathrm{mg} / \mathrm{kg})$, the rabbits were placed on the treatment couch in a prone position, and the tumor tissues were completely immersed into the degassed water. The targeted site of HIFU thermal ablation was guided and monitored using the diagnostic transducer before HIFU exposure. Forty-eight rabbits were randomly divided into three groups: HIFU combined with PBS (PBS group), HIFU combined with SonoVue MBs (MBs group) and HIFU combined with lipid NBs (NBs group), respectively. Each group had sixteen rabbits. In these three groups, the rabbits received an intravenous injection of PBS, SonoVue MBs and lipid NBs solution $\left(0.2 \mathrm{ml} / \mathrm{kg}, 1.0 \times 10^{8}\right.$ bubbles $\left./ \mathrm{ml}\right)$ respectively, and received HIFU exposure after $15 \mathrm{~s}$. During whole experiment process, single "ablated-dot" mode was employed and HIFU exposure parameters were kept the same with an acoustic power at $150 \mathrm{~W}$ and exposure time for $5 \mathrm{~s}$. 
206 Before and after HIFU exposure, the echo intensity of the B-mode ultrasound image within ROI

207

208

209

210

211

212

213

214

215

216

217

218

219

220

221

222

223

224

225

226

227

228

229

230

231

232

233

234

235

236

237

238

239

240

241

242

243

244

245

was also calculated using Gray Val 1.0 Software.

Three days after HIFU exposure, the animals were euthanized with intravenous 3\% pentobarbital $(1.5 \mathrm{ml} / \mathrm{kg})$ and each breast tumor tissue was removed immediately for macroscopic and microscopic examinations. The tissue were cut into $2 \sim 3 \mathrm{~mm}$-thick slices to calculate the volume of the necrotic tissues exposed by HIFU according to the above equation. And then, the tissues were isolated, fixed with formalin, embedded in paraffin. The sections were subsequently stained with hematoxylin and eosin (HE) for pathological examination. In addition, about $1 \mathrm{~mm}^{3}$ necrotic tissue was used to evaluate the ultra-structure changes of cells using transmission electron microscope (TEM).

The immunohistochemical staining was used to detect the tumor cell proliferation (Luo et al., 2007). After being deparaffined in xylene and rehydrated, the sections were blocked with goat serum for $20 \mathrm{~min}$ at room temperature, and then incubated with mouse anti-proliferating cell nuclear antigen (PCNA) monoclonal antibody (diluted 1:500, Boster, Wuhan, China). After rinsing with $\mathrm{PBS}$, the sections were incubated with biotinylated secondary antibody, followed by avidin-biotin peroxidase complex treatment, then counterstained with hematoxylin for $2 \mathrm{~min}$. The PCNA positive cells showed brownish. The PCNA positive index $(\%)=$ the number of PCNA positive cells/total number of cells observed. Five random areas $(400 \times$ magnification $)$ in each slide were observed.

\section{Statistical analysis}

SPSS 20.0 software (SPSS Co., Chicago, USA) was used to analyze the data. All data were expressed as the mean \pm standard deviation. Group differences were analyzed with an independent sample t-test and intragroup comparison was performed using analysis of variance. $P$ value less than 0.05 was considered as a significant difference.

\section{Results}

\section{Characterization of NBs and MBs}

Lipid NBs were successfully prepared with high stability and without morphologic change in PBS solution at room temperature for more than three days. The appearance of lipid NBs suspension was milk white. Under the light microscopy, lipid NBs and SonoVue MBs were small, spherical and distributed evenly (Fig. 1A and B). The mean size of lipid NBs was $565.6 \pm 41.29 \mathrm{~nm}$, and that of SonoVue MBs (SonoVue) was 2429 $\pm 638.6 \mathrm{~nm}$ (Fig. 1C and D). The concentrations of lipid NBs and SonoVue MBs were $(6.12 \pm 0.62) \times 10^{9}$ bubbles $/ \mathrm{ml}$ and $(1.78 \pm 0.22) \times 10^{8}$ bubbles / $\mathrm{ml}$ respectively.

\section{Ex vivo HIFU synergistic effect assessment in excised bovine liver}

After HIFU exposure, the ultrasound image of the targeted area appeared hyperecho (Fig. 2). Under the same HIFU parameters, the quantitative echo intensity of B-mode ultrasound image in lipid NBs groups was obviously higher than that in PBS groups $(* P<0.05)$, but the quantitative echo intensity could not be demonstrated a difference between lipid NBs groups and SonoVue 
246 MBs groups $(* * P>0.05)$ (Fig. 4A). Meanwhile, the echo intensity of targeted area on B-mode 247 ultrasound image after HIFU exposure was increased with the increasing of acoustic power. 248 After HIFU exposure, the volume of necrotic tissues was also measured to compare the 249 difference under diverse HIFU parameters (Fig. 3 and 4B). The coagulative necrosis volume 250 between lipid NBs groups and SonoVue MBs groups could not be demonstrated a difference $251 \quad(* * P>0.05)$, but the coagulative volume in lipid NBs groups was significantly larger than that in 252 PBS groups $(* P<0.05)$. These findings were consistent with the echo intensity results, 253 indicating that lipid NBs could enhance the therapeutic efficiency of HIFU ablation comparable 254 to that of SonoVue MBs.

\section{In vivo HIFU synergistic effect assessment in rabbit breast VX2 tumors}

In this part, we further introduced lipid NBs and SonoVue MBs into rabbit breast VX2 tumor models to evaluate the synergistic effect of lipid NBs for HIFU thermal ablation compared with SonoVue MBs. When introducing lipid NBs and SonoVue MBs, the acoustic signal intensity of the targeted area showed obvious enhancement after HIFU exposure (Fig. 5A-C). The quantitative echo intensity of B-mode ultrasound image showed that the lipid NBs did not show higher synergy compared to that of SonoVue MBs $(* * P>0.05)$, and dramatically higher than that of PBS $\left({ }^{*} P<0.05\right)$ (Fig 7A). After HIFU exposure, the necrotic tissues were different with the ambient tissues on macroscopic inspection, showing that there was a well-defined boundary between them (Fig. 5D- F). The necrotic tissues volume in in vivo tumor models, similarly, revealed that lipid NBs was not superior to that of SonoVue MBs $(* * P>0.05)$, but significantly larger than that of PBS $\left({ }^{*} P<0.05\right)$ (Fig. 7B). After HE staining, there was a sharp demarcation between ablated and non-ablated region in three groups (Fig. 6A-C). In the all necrotic regions, the cells were seen with lysed cell membranes and nuclear fragmentation. In some necrotic regions of PBS group, some cells islets without HIFU ablation remained in the targeted area and arranged in nests (Fig. 6A green arrow). Visualizing by TEM, the structures of cells were ambiguous, and most of cell membranes and nuclear membranes were completely undefined in the three groups (Fig. 6D-F). In the immunohistochemistry examination, the positive index (PI) of PCNA showed no difference between the lipid NBs and SonoVue MBs groups $(* * P>0.05)$, but the PI of PCNA in lipid NBs group was significantly lower than that in PBS group $(* P<0.05) \quad$ (Fig. 6G-I and 7C).

\section{Discussion}

The aim of this paper was to evaluate the synergistic effect of lipid NBs versus SonoVue MBs for HIFU thermal ablation in excised bovine liver and in vivo breast tumor models of rabbits. By carefully comparing the echo intensity of B-mode ultrasound images, the coagulative necrosis volume, and the pathological change after HIFU ablation in the presence of lipid NBs and SonoVue MBs both in ex vivo and in vivo experiments, these results showed that lipid NBs had the same effect as SonoVue MBs for synergistic HIFU thermal ablation. This further indicates that lipid NBs could potentially be used as an enhancer for synergistic HIFU thermal ablation of tumors.

HIFU focuses high-ultrasound-wave energy on the targeted region to produce a tremendous 
287 acoustic pressure, resulting in tissue necrosis due to thermal effect and cavitation effect (Farny, 288 Glynn \& Roy, 2010). However, bubbles in the targeted area could enhance the therapeutic 289 efficiency of HIFU by accelerating ultrasound-mediated heating and lowering cavitation experience nonlinear oscillation known as inertial cavitation, and radiate out of higher frequencies ultrasound with massive energy, which are more readily absorbed by tissues and availably converted into heat to damage the tumor cells (Umemura, Kawabata \& Sasaki, 2005; Holt \& Roy, 2001). So bubbles are an important enhancer for HIFU thermal ablation of tumors. Sokka et al had ever studied endogenous bubbles directly from the tissue to enhance the ultrasound absorption and ultimately create larger lesions in vivo, but this required very high acoustic power and the number and activity of the resultant bubbles was highly variable due to the heterogeneity of tissues (Sokka, King \& Hynynen, 2003). Therefore, exogenous bubbles are potentially an ideal alternative to lower the cavitation threshold, enhance the cavitation activity, and improve the therapeutic efficiency of HIFU thermal ablation.

MBs are well known to be an important enhancer for HIFU thermal ablation by ultrasoundmediated tissue heating and cavitation effect. However, the clinical translation of MBs as an ablation enhancer in HIFU treating tumors is basically limited by its disadvantages. Firstly, MBs are too large to extravasate out of tumor vascular space. Secondly, MBs have a very short blood circulation time. Additionally, excess MBs easily shift the heating position from the targeted area and cause unwanted heating and irreversible thermal damage to healthy tissue (Moyer et al., 2015). With the rapid development of nanotechnology, NBs with various shells present a promising application in disease diagnosis and treatment due to its good imaging ability, long lifetime in blood circulation and strong infiltration out of the endothelial gap of tumors (Yin et al., 2012; Fan et al., 2013). In this study, we compared the synergistic effect of lipid NBs and SonoVue MBs for HIFU thermal ablation of tumor. Between lipid NBs groups and SonoVue MBs groups, the echo intensity within region of interest on B-mode ultrasound images was significantly larger than that of corresponding PBS groups. However, there was not a significant difference between lipid NBs and SonoVue MBs groups both ex vivo and in vivo experiments. In order to further investigate the therapeutic effect of bubbles for enhancing HIFU thermal ablation, we evaluated the coagulative necrosis volume and pathological change after HIFU thermal ablation. These results showed that lipid NBs had the same synergistic effect as SonoVue MBs during HIFU thermal ablation process. Therefore, lipid NBs could potentially become an ideal enhancer of HIFU ablation of tumors.

After HIFU exposure, the appearance of hyperecho of B-mode image at HIFU focus was commonly used to estimate coagulative necrosis of tissue and the volume of necrosis during USguided high intensity focused ultrasound treatment procedure. However, the mechanism of appearance of hyperecho was currently unclear. Rabkin et al. believe that the onset of cavitation had a strong correlation with the appearance of hyperecho at HIFU focus (Rabkin et al., 2005). Their passive cavitation detection results showed that inertial cavitation occurred prior to the appearance of a hyperechoic region on B-mode ultrasound image. Coussions et al. believe that the appearance of hyperechoic regions on B-mode ultrasound image constituted neither a 
necessary nor a sufficient condition for inertial activity to occur during HIFU exposure, but boiling cavities played a significant role in monitoring HIFU therapy as they were readily visible on B-mode ultrasound image (Coussions et al., 2007). In our paper, the echo intensity on Bmode ultrasound image between lipid NBs and SonoVue MBs groups was significantly higher than that of corresponding PBS groups, but it could not demonstrate a difference between lipid NBs and SonoVue MBs groups. We speculated that the hyperecho region was correlated with the production of a mass of bubbles induced by cavitation activity and boiling. Simultaneously, the infusion of lipid NBs or SonVue MBs provided extra bubbles at HIFU focus, which contributed to the production of cavitation bubbles or boiling bubbles. Because of complex and unpredictable of the behavior of acoustic cavitation, the mechanism of between hyperecho on B-mode ultrasound image and acoustic cavitation or boiling need be further studied.

When exposed at high ultrasound pressure, gas-filled bubbles exhibit different destruction mechanisms to biological tissues. Apart from the thermal effect and mechanical action, shock waves, high fluid velocities and free radicals from cavitation also play an important role in lesion formation. When remarkable cavitation is induced in situ, the generated bubbles potentially act as ultrasound scatters and increase ultrasound power deposition in targeted area. Recently, many means were investigated to enhance local heating and cavitation activity during HIFU ablation. The varying components of bubbles' shell membrane and different types of substances inside bubbles have potentially exhibited different efficiencies in tissue heating and cavitation activity. Zhang et al. confirmed lipid-shelled MBs had a greater efficiency than polymer-shelled MBs in heating and cavitation during focused exposures (Zhang et al., 2012). Compared to the hardshelled polymer MBs, the soft-shelled lipid MBs could easily lead to higher harmonics that are more readily absorbed and converted to heating deposition in the targeted area by nonlinear oscillations. However, the small NBs have higher resonant frequency than MBs in the same acoustic field. Whether the small NBs could induce larger lesions than MBs needs further studies using different shelled bubbles and different size of bubbles at high ultrasound pressure. Zhou et al. used uSPIO/PLGA nanoparticles as contrast agents for the enhancement of the effects of HIFU ablation on liver tissue (Zhou et al., 2015). The uSPIO nanoparticles in the shell of the microspheres could boost the acoustic impedance to generate stronger ultrasound scattering and improve heating deposition in the targeted area. Compared to uSPIO nanoparticles, gas-filled bubbles could exhibit stronger acoustic impedance difference between bubbles and surrounding biological tissues, which more easily lead to heating deposition in the targeted area. The shelled components of NBs used in this work were similar with that of SonoVue MBs, and the gas core was different. Thus more studies will be needed to elucidate the influence of components of bubbles in high pressure ultrasound field and promote the clinical translation.

In addition to enlarging the tissue lesions, bubbles could reduce the occurrence of residual tumor during HIFU thermal ablation. Pathological inspections in this paper revealed that there were no non-ablated cells within the targeted area in both lipid NBs group and SonoVue MBs group, but one observation worth noting is that there were still some non-ablated cells within some targeted area in the PBS group (Fig. 6A green arrow). These findings have been reported in some previous literatures (Orsi et al., 2015; Boutier et al., 2011). This study also showed the 
synergistic therapy of bubbles for HIFU thermal ablation can effectively reduce the residual tumor in the targeted area.

The thermal effect and cavitation activity play an important direct role in killing tumor cells during HIFU ablation. Recently, however some studies showed that HIFU also has a potential to induce the whole body antitumor immune response for effective tumor therapy (Unga \& Hashida, 2014). HIFU destruction of tumors may lead to immunity forming in the body by infiltration of immune cells into the tumor and exposure of antigen. Some scholars demonstrated that the formation of cell debris generated by cavitation activity and mechanical effect of HIFU was more beneficial to activation of the whole body antitumor immune response, rather than coagulation necrosis come from thermal effect of HIFU (Hu et al., 2007; Wu et al., 2004).. In our immunohistochemical examination, the positive index of PCNA was obviously reduced, especially in both lipid NBs group and SonoVue MBs group. It well reflected that HIFU exposure contributed to suppress the proliferation of tumor cells, particularly in the presence of bubbles. In addition, lysed cell membranes and nuclear fragmentation contributed to activate the immune response. But we did not try to detect and analyze the antitumor immune response in the present study. Therefore, the exact mechanism of antitumor immune response induced by bubbles in the acoustic field needs further investigation.

Several limitations of our study should be addressed. First, the biocompatibility, biodistribution and biosecurity of lipid NBs in vivo are not shown in our study. Second, we did not explore the temperature change caused by the bubbles in this study. Third, we did not directly investigate the enhanced permeability and retention (EPR) effect of NBs in the tumor. Finally, we just employed single "ablated-dot" mode in HIFU thermal ablation of rabbit breast VX2 tumors. The effect of the whole tumor ablation by HIFU combined with lipid NBs should be further addressed.

\section{Conclusions}

In this study, we introduced lipid NBs and SonoVue MBs into the targeted area of HIFU thermal ablation and explored the synergistic effect of lipid NBs and SonoVue MBs for HIFU thermal ablation in ex vivo bovine liver and in vivo breast tumor models of rabbits. By analysis of the echo intensity change of B-mode image after HIFU thermal ablation, the volume of necrotic tissues, macroscopic and microscopic examinations of necrotic tissue and immunohistochemical examination of PCNA of tumor cells, these results showed that lipid NBs had the same effect as SonoVue MBs for synergistic HIFU thermal ablation. All in all, our study suggested that lipid NBs had the same effect as SonoVue MBs for synergistic HIFU thermal ablation with similar shell materials. In conclusion, lipid NBs are not only a good contrast agent for ultrasound molecular imaging and a fine vector for drug delivery and gene transfection, but also an potentially enhancer for HIFU thermal ablation of tumors.

\section{Acknowledgments}

We would like to thank Chongyan Li for affording the VX2 tumor tissue, and Dan Zhou and Fenfen $\mathrm{Xu}$ for performing excised bovine liver experiment. 
410

411

412

413

414

415

416

417

418

419

420

421

422

423

424

425

426

427

428

429

430

431

432

433

434

435

436

437

438

439

440

441

442

443

444

445

446

447

448

449

450

\section{Author Declaration}

There is no conflict of interest for publication.

\section{References}

Hassanuddin A, Choi JH, Seo DW, Ryu CH, Kim SH, Park do H, Lee SS, Lee SK, and Kim MH. 2014. Factors affecting tumor ablation during high intensity focused ultrasound treatment. Gut and Liver 8:433-437. DOI 10.5009/gnl.2014.8.4.433.

Peek MC, Ahmed M, Napoli A, ten Haken B, McWilliams S, Usiskin SI, Pinder SE, van Hemelrijck M, and Douek M. 2015. Systematic review of high-intensity focused ultrasound ablation in the treatment of breast cancer. British Journal of Surgery 102:873-882. DOI 10.1002/bjs.9793.

Zhang M, Fabiilli ML, Haworth KJ, Padilla F, Swanson SD, Kripfgans OD, Carson PL, and Fowlkes JB. 2011. Acoustic droplet vaporization for enhancement of thermal ablation by high intensity focused ultrasound. academic radiology 18:1123-1132. DOI 10.1016/j.acra.2011.04.012.

Cavallo Marincola B, Pediconi F, Anzidei M, Miglio E, Di Mare L, Telesca M, Mancini M, D'Amati G, Monti M, Catalano C, and Napoli A. 2015. High-intensity focused ultrasound in breast pathology: non-invasive treatment of benign and malignant lesions. expert review of medical devices 12:191-199. DOI 10.1586/17434440.2015.986096.

Uchida T, Nakano M, Hongo S, Shoji S, Nagata Y, Satoh T, Baba S, Usui Y, and Terachi T. 2012. High-intensity focused ultrasound therapy for prostate cancer. international journal of urology 19:187-201. DOI 10.1111/j.1442-2042.2011.02936.x.

Kazarian AM, Anchikov G, Khol PK, Fosse E, Edvin B, and Grachev SV. 2008. High-intensity focused ultrasound ablation, a new method for the minimally invasive treatment of hepatic tumours. Vestn Ross Akad Med Nauk: 63-68. DOI 10.1097/01.bco.0000183682.

Hesley G K, Gorny K R and Woodrum D A. 2013. MR-guided focused ultrasound for the treatment of uterine fibroids. Cardiovasc Intervent Radiol 36:5-13. DOI 10.1007/s00270012-0367-3.

Zhou YF. 2011. High intensity focused ultrasound in clinical tumor ablation. World Journal of Clinical Oncology 2:8-27. DOI 10.5306/wjco.v2.i1.8.

Fischer K, Gedroyc W, and Jolesz FA. 2010. Focused ultrasound as a local therapy for liver cancer. Cancer J 16:118-124. DOI 10.1097/PPO.0b013e3181db7c32.

Ellens NP, Lucht BB, Gunaseelan ST, Hudson JM, and Hynynen KH. 2015. A novel, flat, electronically-steered phased array transducer for tissue ablation: preliminary results. Phys Med Biol 60:2195-2215. DOI 10.1088/0031-9155/60/6/2195.

Moyer LC, Timbie KF, Sheeran PS, Price RJ, Miller GW, and Dayton PA. 2015. High-intensity focused ultrasound ablation enhancement in vivo via phase-shift nanodroplets compared to microbubbles. Journal of Therapeutic Ultrasound 3:7. DOI 10.1186/s40349-015-0029-4.

Sun Y, Zheng Y, Ran H, Zhou Y, Shen H, Chen Y, Chen H, Krupka TM, Li A, Li P, Wang Z, and Wang Z. 2012. Superparamagnetic PLGA-iron oxide microcapsules for dual-modality US/MR imaging and high intensity focused US breast cancer ablation. Biomaterials 
451

452

453

454

455

456

457

458

459

460

461

462

33:5854-5864. DOI 10.1016/j.biomaterials.2012.04.062.

Hamano N, Negishi Y, Takatori K, Endo-Takahashi Y, Suzuki R, Maruyama K, Niidome T, and Aramaki Y. 2014. Combination of bubble liposomes and high-intensity focused ultrasound (HIFU) enhanced antitumor effect by tumor ablation. Biological \& Pharmaceutical Bulletin 37:174-177. DOI 10.1248/bpb.b13-00605.

Ma M, Xu H, Chen H, Jia X, Zhang K, Wang Q, Zheng S, Wu R, Yao M, Cai X, Li F, and Shi J. 2014. A drug-perfluorocarbon nanoemulsion with an ultrathin silica coating for the synergistic effect of chemotherapy and ablation by high-intensity focused ultrasound. Advanced Materials 26:7378-7385. DOI 10.1002/adma.201402969.

Zhang X, Zheng Y, Wang Z, Huang S, Chen Y, Jiang W, Zhang H, Ding M, Li Q, Xiao X, Luo $X$, Wang Z, and Qi H. 2014. Methotrexate-loaded PLGA nanobubbles for ultrasound imaging and Synergistic Targeted therapy of residual tumor during HIFU ablation. Biomaterials 35:5148-5161. DOI 10.1016/j.biomaterials.2014.02.036.

Zhou D, Sun Y, Zheng YY, Ran HT, Li P, Wang ZB, and Wang ZG. 2015. Superparamagnetic PLGA-iron oxide microspheres as contrast agents for dual-imaging and the enhancement of the effect of high-intensity focused ultrasound ablation on liver tissue. RSC Advances 5:35693-35703. DOI 10.1039/C5RA00880H.

Luo W, Zhou X, Tian X, Ren X, Zheng M, Gu K, and He G. 2006. Enhancement of ultrasound contrast agent in high-intensity focused ultrasound ablation. Advances in Therapy 23:861868. DOI 10.1007/BF02850207.

Peng S, Xiong Y, Li K, He M, Deng Y, Chen L, Zou M, Chen W, Wang Z, He J, and Zhang L. 2012. Clinical utility of a microbubble-enhancing contrast ("SonoVue") in treatment of uterine fibroids with high intensity focused ultrasound: a retrospective study. European Journal of Radiology 81:3832-3838. DOI 10.1016/j.ejrad.2012.04.030.

Wu F, Wang ZB, Lu P, Xu ZL, Chen WZ, Zhu H, and Jin CB. 2004. Activated anti-tumor immunity in cancer patients after high intensity focused ultrasound ablation. Ultrasound in Medicine \& Biology 30:1217-1222. DOI 10.1016/j.ultrasmedbio.2004.08.003.

Liu HL, Hsieh HY, Lu LA, Kang CW, Wu MF, and Lin CT. 2012. Low-pressure pulsed focused ultrasound with microbubbles promotes an anticancer immunological response. Journal of Translational Medicine 10:221. DOI 10.1186/1479-5876-10-221.

Coussios CC, Farny CH, Haar GT, and Roy RA. 2007. Role of acoustic cavitation in the delivery and monitoring of cancer treatment by high-intensity focused ultrasound (HIFU). International Journal of Hyperthermia 23:105-120. DOI 10.1080/02656730701194131.

Tran BC, Seo J, Hall TL, Fowlkes JB, and Cain CA. 2005. Effect of contrast agent infusion rates on thresholds for tissue damage produced by single exposure of high-intensity ultrasound. IEEE Transactions on Ultrasonics Ferroelectrics and Frequency Control 52:1121-1130. DOI 10.1109/TUFFC.2005.1503998.

McDannold NJ, Vykhodtseva NI, and Hynynen K. 2006. Microbubble contrast agent with focused ultrasound to create brain lesions at low power levels: MR imaging and histologic study in rabbits. Radiology 241:95-106. DOI 10.1148/radiol.2411051170.

Kopechek JA, Park EJ, Zhang YZ, Vykhodtseva NI, McDannold NJ, and Porter TM. 2014. 
492

493

494

495

496

497

Cavitation-enhanced MR-guided focused ultrasound ablation of rabbit tumors in vivo using phase shift nanoemulsions. Physics in Medicine and Biology 59:3465-3481. DOI 10.1088/0031-9155/59/13/3465

Zhang M, Fabiilli ML, Haworth KJ, Padilla F, Swanson SD, Kripfgans OD, Carson PL, and Fowlkes JB. 2011. Acoustic droplet vaporization for enhancement of thermal ablation by high intensity focused ultrasound. Academic Radiology 18:1123-1132. DOI 10.1016/j.acra.2011.04.012.

Rapoport N, Nam KH, Gupta R, Gao Z, Mohan P, Payne A, Todd N, Liu X, Kim T, Shea J, Scaife C, Parker DL, Jeong EK, and Kennedy AM. 2011. Ultrasound-mediated tumor imaging and nanotherapy using drug loaded, block copolymer stabilized perfluorocarbon nanoemulsions. Journal of Controlled release 153:4-15. DOI 10.1016/jconrel.2011.01.022. Huang HY, Hu SH, Hung SY, Chiang CS, Liu HL, Chiu TL, Lai HY, Chen YY, and Chen SY. 2013. SPIO nanoparticle-stabilized PAA-F127 thermosensitive nanobubbles with MR/US dual-modality imaging and HIFU-triggered drug release for magnetically guided in vivo tumor therapy. Journal of Controlled Release 172:118-127. DOI 10.1016/j.jconrel.2013.07.029.

Yin T, Wang P, Zheng R, Zheng B, Cheng D, Zhang X, and Shuai X. 2012. Nanobubbles for enhanced ultrasound imaging of tumors. International Journal of Nanomedicine 7:895-904. DOI 10.2147/IJN.S28830.

Fan X, Wang L, Guo Y, Tong H, Li L, Ding J, and Huang H. 2013. Experimental investigation of the penetration of ultrasound nanobubbles in a gastric cancer xenograft. Nanotechnology 24:325102. DOI 10.1088/0957-4484/24/32/325102.

Xie X, Lin W, Liu H, Deng J, Chen Y, Liu H, Fu X, and Yang Y. 2015. Ultrasound-responsive nanobubbles contained with peptide-camptothecin conjugates for targeted drug delivery. Drug Delivery:1-9. DOI 10.3109/10717544.2015.1077289.

Cavalli R, Bisazza A, and Lembo D. 2013. Micro- and nanobubbles: a versatile non-viral platform for gene delivery. International Journal of Pharmaceutics 456:437-445. DOI 10.1016/j.ijpharm.2013.08.041.

Wang D, Li L, Min J, Guo Y, Guo H, Gao J, Yang K, and Zou J. 2012. Combination of highintensity focused ultrasound with nanoscale ultrasound contrast agent in treatment of rabbit breast VX2 tumors: a pilot study. Clinical Imaging 36:717-723. DOI 10.1016/j.clinimag.2012.01.042.

Wang D, Yang K, Gao YH, Tan KB, and Liu Z. 2010. Preparation and characterization of a nanoscale ultrasound contrast agent. Clinical Imaging 34:288-292. DOI 10.1016/j.clinimag.2010.02.009.

Luo W, Zhou XD, Zhang J, Qian YQ, Zheng MJ, Yu M, and Gong XY. 2007. Analysis of apoptosis and cell proliferation after high intensity-focused ultrasound ablation combined with microbubbles in rabbit livers. European Journal of Gastroenterology \& Hepatology 19:962-968. DOI 10.1097/MEG.0b013e3282cfb6f0.

Farny CH, Glynn HR, and Roy RA. 2010. The correlation between bubble-enhanced HIFU heating and cavitation power. IEEE Transations on Biomedical Engineering 57:175-184. 
533

534

535

536

537

538

539

540

541

542

543

544

545

546

547

548

549

550

551

552

553

554

555

556

557

558

559

560

561

562

563

564

565

566

567

568

569

DOI 10.1109/TBME.2009.2028133.

Tung YS, Liu HL, Wu CC, Ju KC, Chen WS, and Lin WL. 2006. Contrast-agent-enhanced ultrasound thermal ablation. Ultrasound in Medicine \& Biology 32:1103-1110. DOI 10.1016/j.ultrasmedbio.2006.04.005.

Kaneko Y, Maruyama T, Takegami K, Watanebbe T, Mitsui H, Hanajiri K, Nagawa H, and Matsumoto Y. 2005. Use of a microbubble agent to increase the effects of high intensity focused ultrasound on liver tissue. European Radiology 15:1415-1420. DOI 10.1007/s00330-005-2663-7.

Umemura S, Kawabata K, and Sasaki K. 2005. In vivo acceleration of ultrasonic tissue heating by microbubble agent. IEEE Transactions on Ultrasonics, Ferroelectrics and Frequency Control 52:1690-1698. DOI 10.1109/TUFFC.2005.1561623.

Holt RG, and Roy RA. 2001. Measurements of bubble-enhanced heating from focused, MHzfrequency ultrasound in a tissue-mimicking material. Ultrasound in Medicine \& Biology 27:1399-1412. DOI 10.1016/S0301-5629(01)00438-0.

Sokka SD, King R, and Hynynen K. 2003. MRI-guided gas bubble enhanced ultrasound heating in in vivo rabbit thigh. Physics in Medicine and Biology 48: 223-241. DOI 10.1088/00319155/48/2/306.

Rabkin BA, Zderic V, and Vaezy S. 2005. Hyperechoic ultrasound images of HIFU therapy: involvement of cavitation. Ultrasound Med Biol 31:947-956. DOI 10.1016/j.ultrasmedbio.2005 03.015.

Zhang SY, Zong YJ, Wan MX, Yu XJ, Fu QY, Ding T, Zhou FY, and Wang SP. 2012. Compare ultrasound-mediated heating and cavitation between flowing polymer- and lipid-shelled microbubbles during focused ultrasound exposure. Journal of Acoustical Socienty of America 131: 4845-4855. DOI 10.1121/1.4714339.

Orsi F, Monfardini L, Bonomo G, Krokidis M, Della Vigna P, and Disalvatore D. 2015. Ultrasound guided high intensity focused ultrasound (USgHIFU) ablation for uterine fibroids: Do we need the microbubbles? international journal of hyperthermia 31:233-239. DOI 10.3109/02656736.2015.1004134.

Boutier R, Girouin N, Cheikh AB, Belot A, Rabilloud M, Gelet A, Chapelon JY, and Rouviere O. 2011. Location of residual cancer after transrectal high-intensity focused ultrasound ablation for clinically localized prostate cancer. BJU International 108:1776-1781. DOI 10.1111/j.1464-410X.2011.10251.x.

Unga J, and Hashida M. 2014. Ultrasound induced cancer immunotherapy. Advanced Drug Delivery Reviews 72:144-153. DOI 10.1016/j.addr.2014.03.004.

Hu Z, Yang XY, Liu Y, Sankin GN, Pua EC, Morse MA, Lyerly HK, Clay TM, and Zhong P. 2007. Investigation of HIFU-induced anti-tumor immunity in a murine tumor model. Journal of Translational Medicine 5:34. DOI 10.1186/1479-5876-5-34. 


\section{1}

characterization of lipid NBs and SonoVue MBs

(A and B) Optical images of lipid NBs and SonoVue MBs in the microscope; (C and D) The size distribution of lipid NBs and SonoVue MBs by dynamic light scattering measurement.
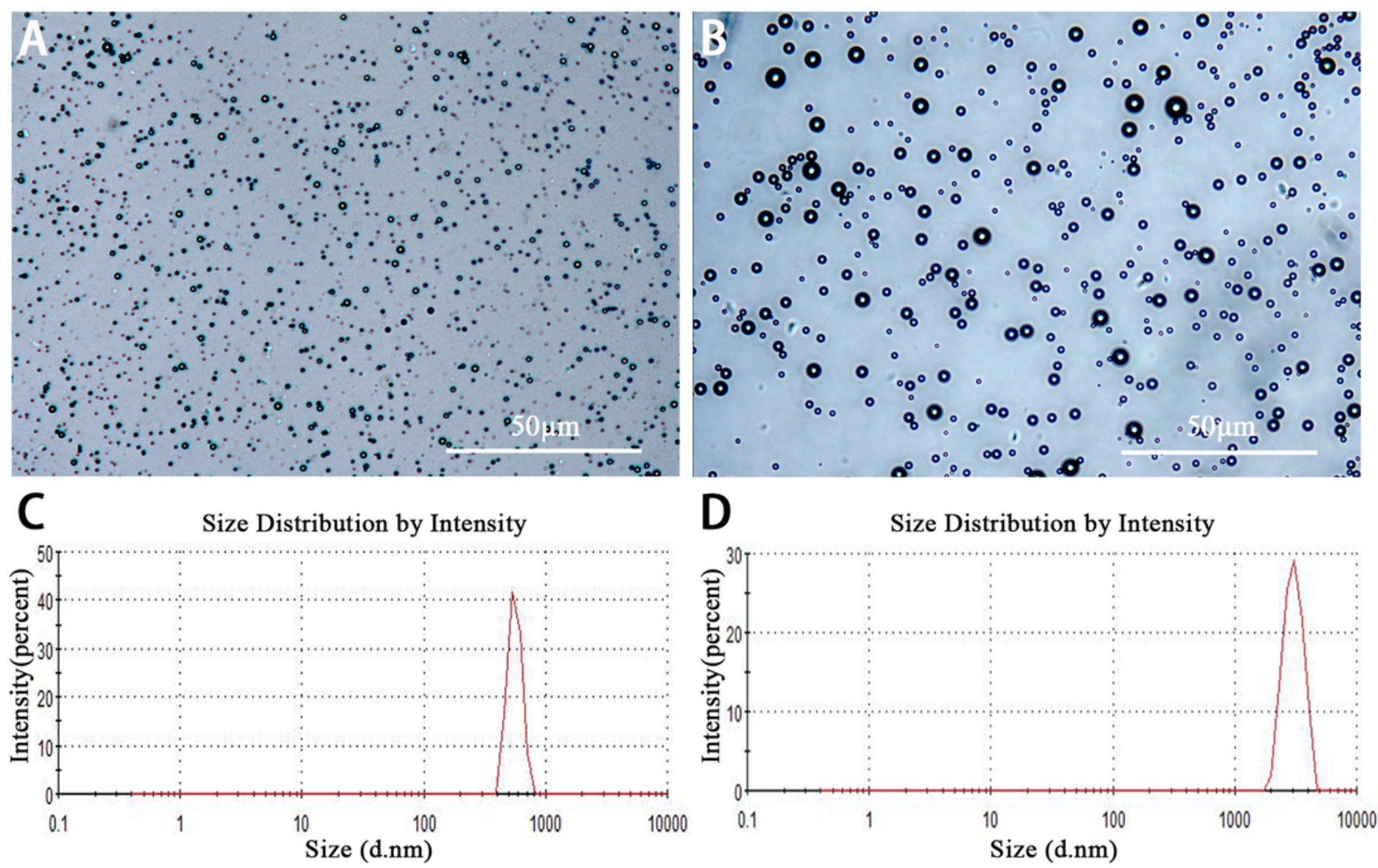


\section{2}

ultrasonoscopy of the targeted area in excised bovine liver before and after HIFU ablation

$\left(A_{1}, B_{1}, C_{1}, D_{1}, E_{1}, F_{1}, G_{1}, H_{1}, l_{1}\right)$ Ultrasonoscopy of the targeted area in excised bovine liver before HIFU ablation. $\left(A_{2}, B_{2}, C_{2}, D_{2}, E_{2}, F_{2}, G_{2}, H_{2}, I_{2}\right)$ Ultrasonoscopy of the targeted area in the excised bovine liver after HIFU ablation with $200 \mu$ PBS, SonoVue MBs and lipid NBs with concentration of $1.0 \times 10^{5}$ bubbles $/ \mathrm{ml}$ at different acoustic power (120W for $5 \mathrm{~s} ; 150 \mathrm{~W}$ for $5 \mathrm{~s}$ and $180 \mathrm{~W}$ for $5 \mathrm{~s}$ ). After HIFU ablation, the echo intensity of the targeted area (green mark) was significantly enhanced.

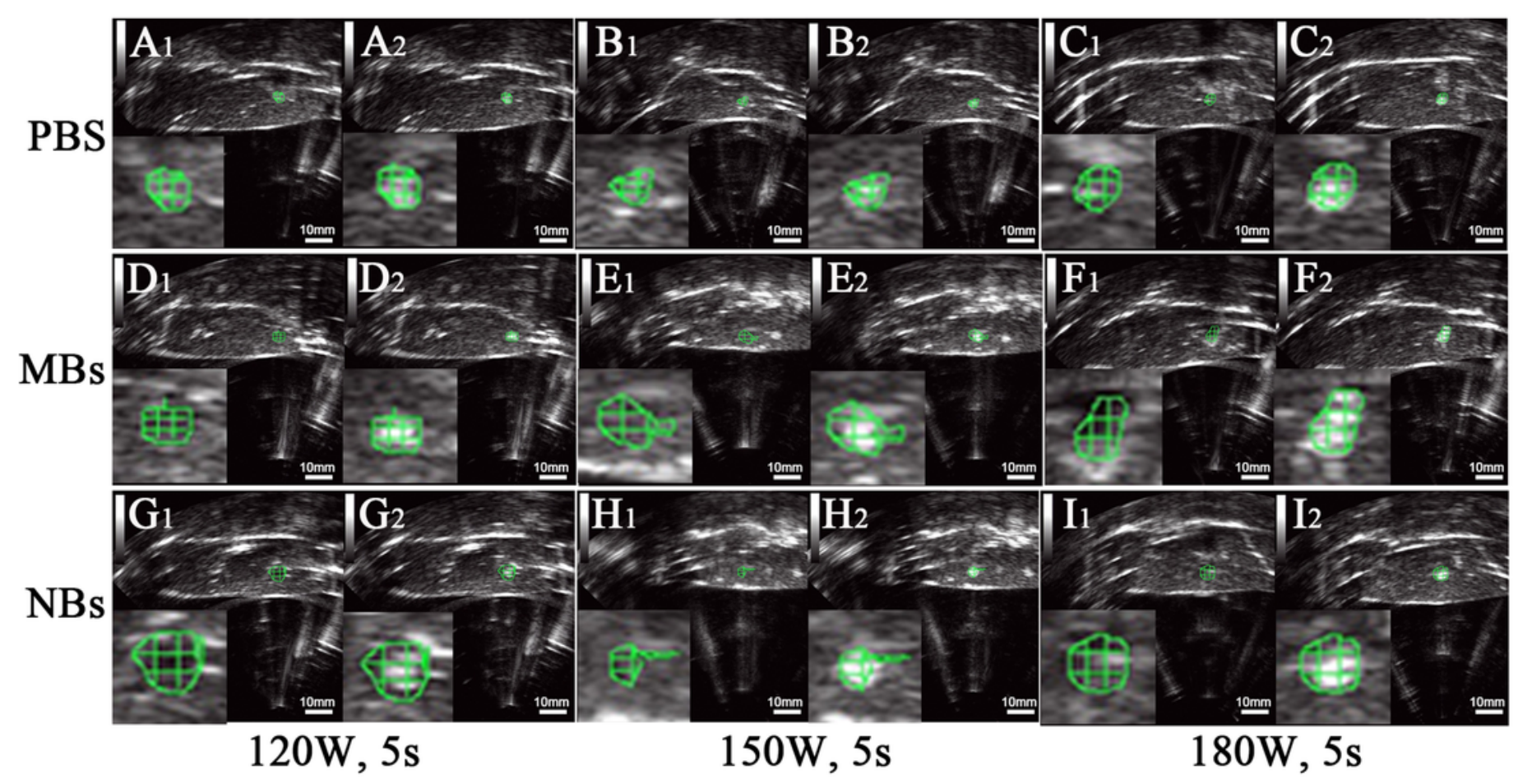


3

Photography of the targeted area in excised bovine liver after HIFU ablation.

After HIFU ablation, the targeted area showed grey white in color. The boundary against the surrounding tissue was sharp and clear. 


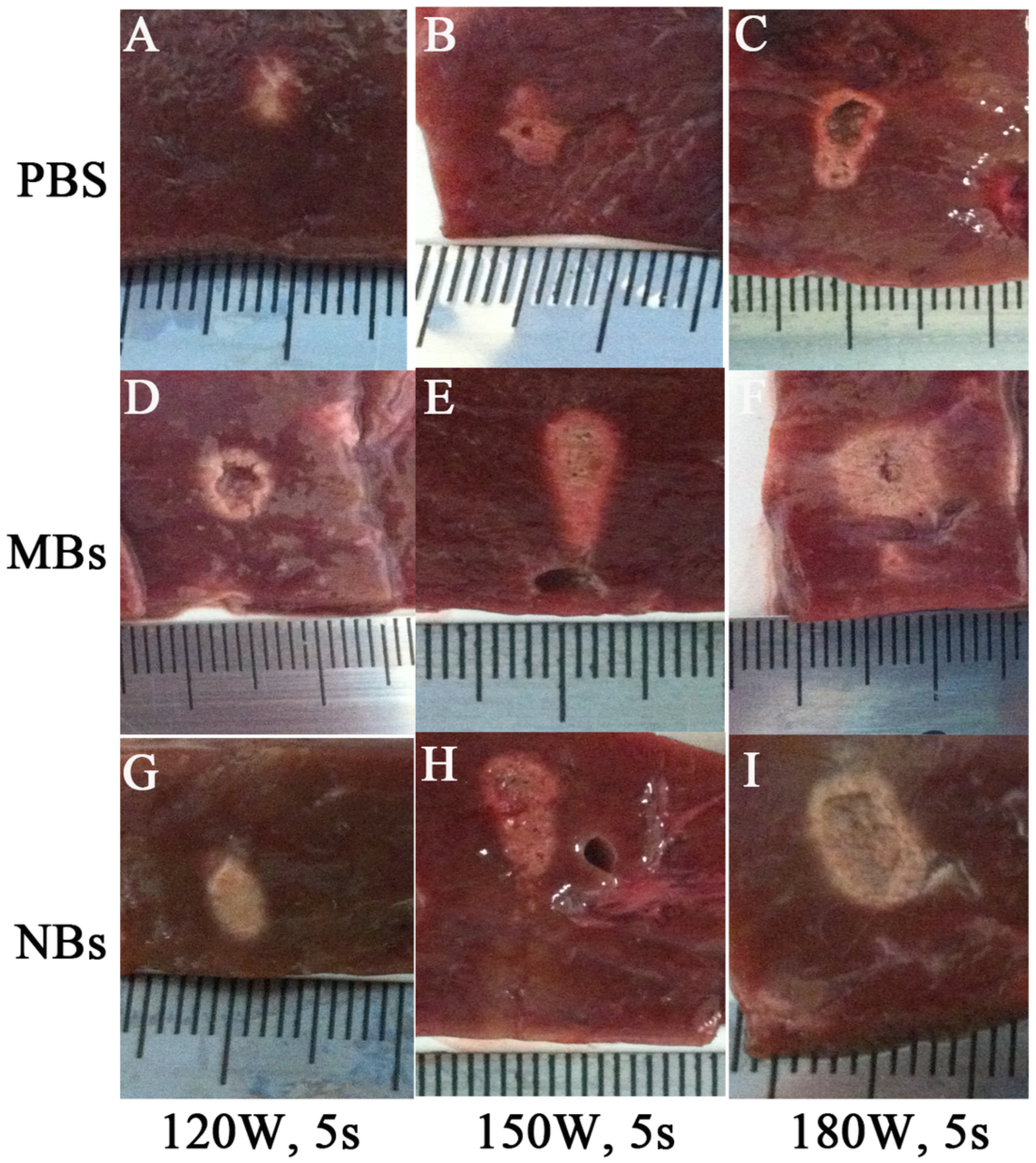


4

Quantitative analysis of echo intensity and coagulative necrosis volume of the targeted area after HIFU ablation.

(A) Quantitative analysis of echo intensity and (B) quantitative analysis of coagulative necrosis volume of the targeted area in the excised bovine liver after HIFU ablation. ${ }^{*} P<$ $0.05 . * * P>0.05$.
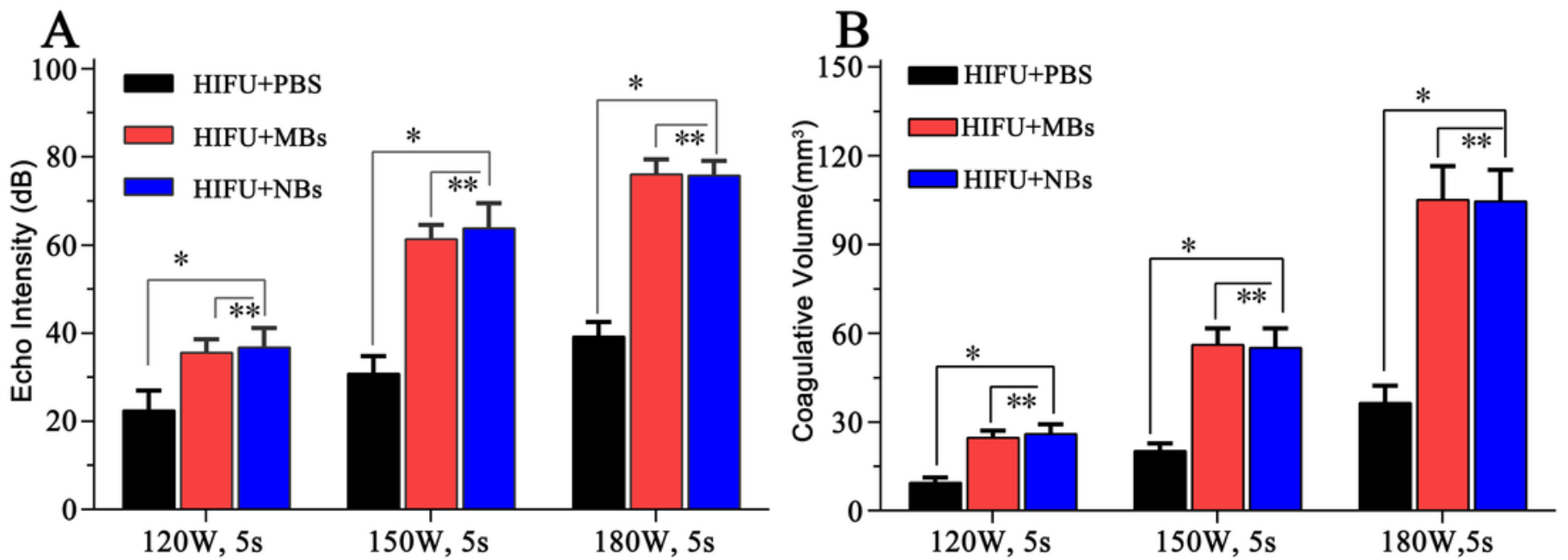


\section{5}

Ultrasonoscopy and photography of the targeted area in the rabbit breast tumor after HIFU ablation.

Ultrasonoscopy showed echoes of the targeted area in rabbit breast tumor before $\left(A_{1}, B_{1}, C_{1}\right)$ and after $\left(\mathrm{A}_{2}, \mathrm{~B}_{2}, \mathrm{C}_{2}\right)$ HIFU ablation. (A) HIFU + PBS; (B) HIFU+ SonoVue MBs; (C) HIFU + lipid NBs. The green mark shows the range of tumor. (D, E, F) Photography of the targeted area in rabbit breast tumor after HIFU ablation combined with PBS, SonoVue MBs and lipid NBs, respectively. The necrotic tissue showed gray (white arrow) and non-ablated tissue appeared darkled (black arrow).
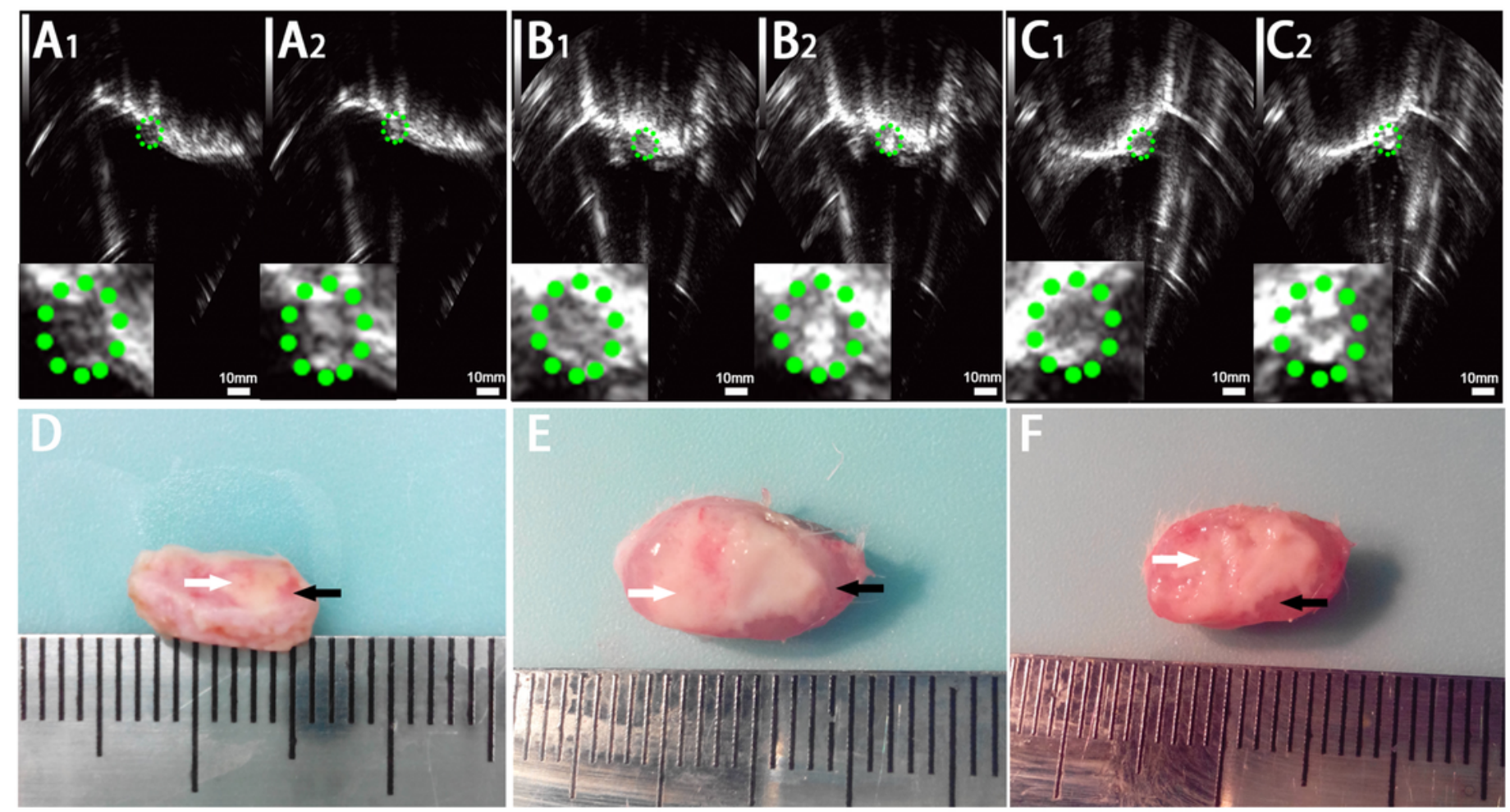
6

Pathological examination of the targeted area in rabbit breast tumor after HIFU ablation.

(A, B , C) HE staining of the targeted area after HIFU ablation (200 xmagnification). A sharp demarcation was showed between ablated (white arrow) and non-ablated (black arrow) region. The green arrow showed the residual tumor cells in the targeted area in PBS group. (D, E, F) TEM photos of the targeted tissue after HIFU ablation. Cell membranes (black arrow) and nucear membranes (white arrow) were interrupted and indefinable. ( $G, H, I)$ Expression of PCNA in tumor tissue after HIFU ablation (200xmagnification). The brown (yellow arrow) indicated PCNA-positive cells, the blue indicated PCNA-negative cells (green arrow). (A, D, G) HIFU + PBS; $(B, E, H)$ HIFU + SonoVue MBs; $(C, F, I) H I F U$ + NBs.

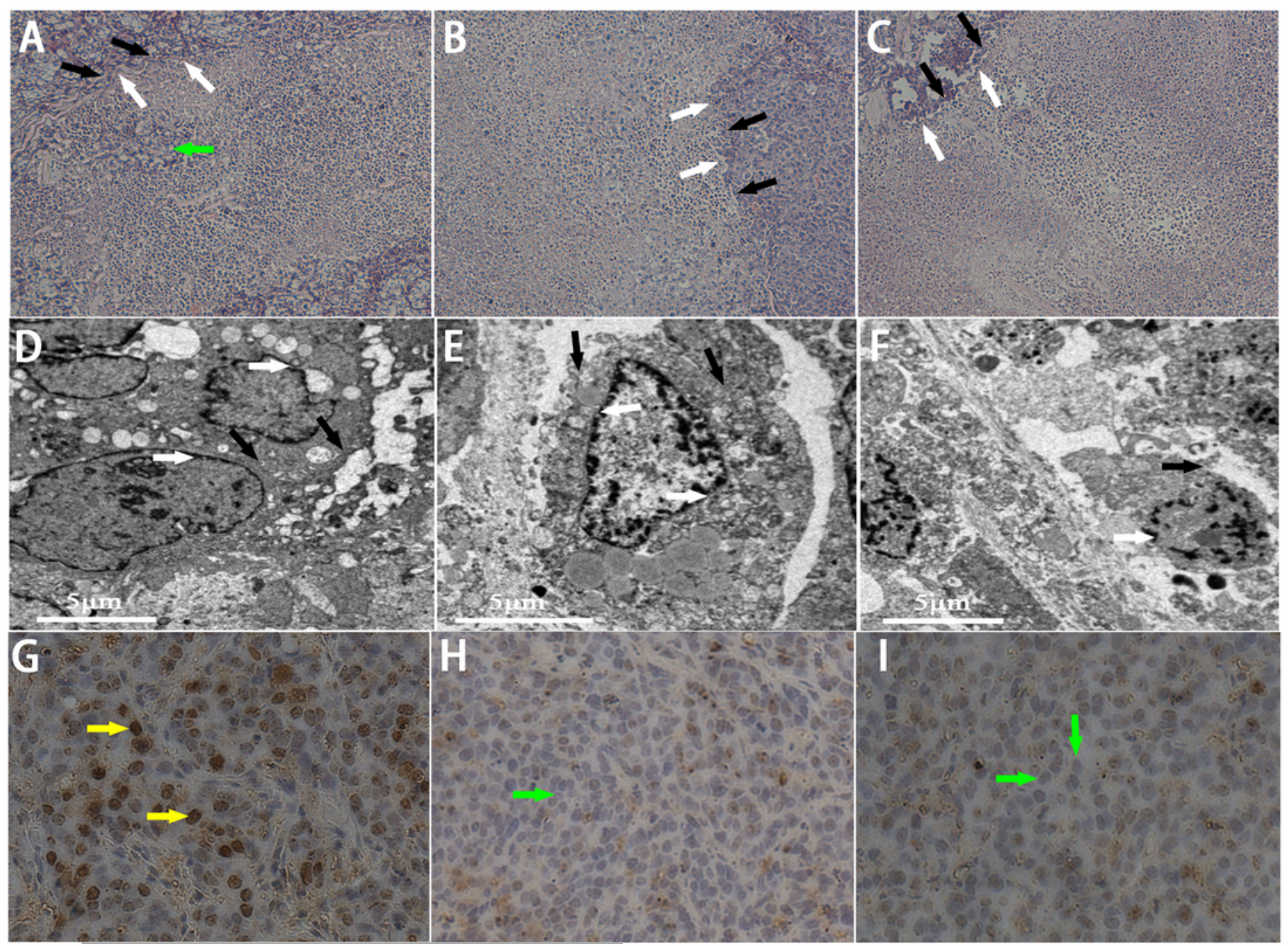


7

Quantitative analysis of echo intensity, coagulative necrosis volume and PCNA positive index in tumor tissue after HIFU ablation.

(A) Quantitative analysis of echo intensity, (B) Quantitative analysis of coagulative necrosis volume and (C) PCNA positive index in tumor tissue after HIFU ablation in HIFU + PBS, HIFU + SonoVue MBs and HIFU + lipid NBs groups. $* P<0.05 ; * * P>0.05$.
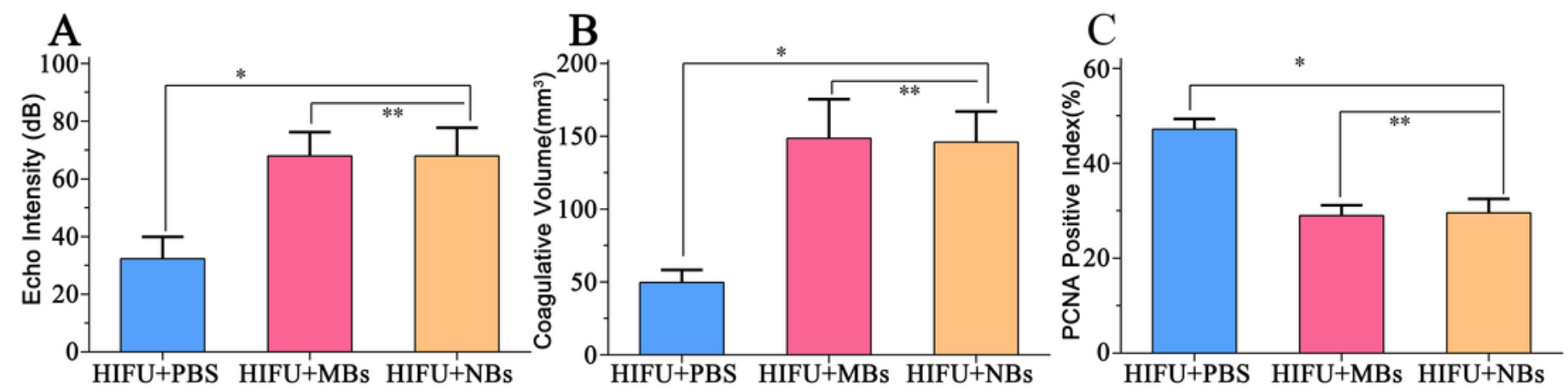